Numer. Math. (1997) 78: 189-209

Numerische Mathematik

\title{
Conditioning analysis of block incomplete factorizations and its application to elliptic equations
}

\author{
Hao Lu ${ }^{1}$, Owe Axelsson ${ }^{2}$ \\ 1 Department of Applied Mathematics, University of Twente, P.O. Box 217, 7500 AE Enschede, \\ The Netherlands \\ 2 Department of Mathematics, University of Nijmegen, Toernooiveld, 6525 ED Nijmegen, \\ The Netherlands
}

Received March 27, 1996 / Revised version received December 27, 1996

Summary. The paper deals with eigenvalue estimates for block incomplete factorization methods for symmetric matrices. First, some previous results on upper bounds for the maximum eigenvalue of preconditioned matrices are generalized to each eigenvalue. Second, upper bounds for the maximum eigenvalue of the preconditioned matrix are further estimated, which presents a substantial improvement of earlier results. Finally, the results are used to estimate bounds for every eigenvalue of the preconditioned matrices, in particular, for the maximum eigenvalue, when a modified block incomplete factorization is used to solve an elliptic equation with variable coefficients in two dimensions. The analysis yields a new upper bound of type $\gamma h^{-1}$ for the condition number of the preconditioned matrix and shows clearly how the coefficients of the differential equation influence the positive constant $\gamma$.

Mathematics Subject Classification (1991): 65F10, 65F15, 65F50

\section{Introduction}

Let $A$ be a symmetric matrix and let

$$
C=(X+L) X^{-1}\left(X+L^{\mathrm{T}}\right),
$$

where $X$ is symmetric positive definite or, briefly s.p.d., and $L$ is a real matrix. Consider solving linear systems $A \mathbf{x}=\mathbf{b}$. A block incomplete factorization of $A$ is of the form (1) with a block diagonal matrix $X$ and a strictly lower block triangular matrix $L$. General approaches of block incomplete factorizations were presented in [12] and [5]. To estimate the rate of convergence of preconditioned iterative methods such as the Chebyshev and the conjugate gradient methods we need to know the distribution of eigenvalues or, at least, the bounds of the extreme eigenvalues of the preconditioned matrices, see $[1,2,6,14,16,23]$. In 
the past decade eigenvalue estimates for block incomplete factorization methods have been extensively studied for symmetric positive definite matrices, see $[3,4$, $8-11,13,17-21]$. Recently, in [7] the authors unified some previous results and obtained some strong results even when the assumptions of $A$, made previously in $[11,18,21]$, were weakened.

For symmetric matrices $B$ and $D$, throughout the paper $B \geq D$ means that $B-D$ is positive semidefinite, and $\lambda_{i}(A)$ denotes the $i$ th eigenvalue of $A$. The eigenvalues of a matrix are ordered in a non-increasing order. Let $A, X$ and $L$ be partitioned in blocks according to a given partitioning, and let $m \times m$ be the number of matrix blocks.

The fundamental result in [7] is that

$$
\lambda_{i}(M(\beta)) \leq \lambda_{i}\left(C^{-1} A\right) \leq \lambda_{i}(M(\sigma)),
$$

where $M(\alpha)=(I+\widetilde{L})^{-1}+\left(I+\widetilde{L}^{\mathrm{T}}\right)^{-1}+(\alpha-2)(I+\widetilde{L})^{-1}\left(I+\widetilde{L}^{\mathrm{T}}\right)^{-1}, \widetilde{L}=X^{-\frac{1}{2}} L X^{-\frac{1}{2}}, \sigma$ and $\beta$ are constant such that $\beta X+L+L^{\mathrm{T}} \leq A \leq \sigma X+L+L^{\mathrm{T}}$. Applying this result to block incomplete factorizations, we obtained some simple upper bounds for the maximum eigenvalue of the preconditioned matrix, for example, $1 /(2-\sigma)$ if $\sigma<2$ and $2 m$ if $\sigma \leq 2$ and $A$ is positive semidefinite.

This paper continues our analysis of block incomplete factorization methods. In order to derive a bound for the condition number of the preconditioned matrix for modified block factorizations we emphasize the upper bound of eigenvalues in the present paper. However, some of the approaches are also applicable to estimates of lower bounds for the preconditioned matrix. First we show that if there exist two constants $\alpha_{i}$ and $\sigma_{i}$ such that $\alpha_{i} \leq \lambda_{i}\left(X^{-1} K\right) \leq \sigma_{i}$, where $K=A-L-L^{\mathrm{T}}$, then

$$
\lambda_{\min }\left(M\left(\alpha_{i}\right)\right) \leq \lambda_{i}\left(C^{-1} A\right) \leq \lambda_{\max }\left(M\left(\sigma_{i}\right)\right) .
$$

Note that $\sigma_{i}$ are also ordered in a non-increasing order. The result yields immediately a simple upper bound $1 /\left(2-\sigma_{i}\right)$ for the $i$ th eigenvalue of the preconditioned matrix $C^{-1} A$ if $\sigma_{i}<2$, which result generalizes the old one for the maximum eigenvalue. Then upper bounds for the maximum eigenvalue are further studied. We derive several upper bounds for the maximum eigenvalue of the preconditioned matrix involving all $\sigma_{i}$, which presents a substantial improvement over the previous results on this topic. One of the results shows immediately that $m$ is another upper bound for the maximum eigenvalue of $C^{-1} A$ if $\sigma_{1} \leq 2$ and $C \leq A$. The approaches are also applicable to every eigenvalue. Finally, the results are used to estimate upper bounds for the maximum eigenvalue of the preconditioned matrix if a modified block incomplete factorization method is used to solve an elliptic equation

$$
\begin{gathered}
-\frac{\partial}{\partial x}\left(a_{1} \frac{\partial}{\partial x} u\right)-\frac{\partial}{\partial y}\left(a_{2} \frac{\partial}{\partial y} u\right)=f \quad \text { on } \Omega, \\
u=g \text { on } \Gamma_{0} \\
\frac{\partial u}{\partial n}=k \text { on } \Gamma_{1}=\partial \Omega-\Gamma_{0},
\end{gathered}
$$


where $\Omega=(0, a) \times(0, b)$, and the coefficients $a_{1}$ and $a_{2}$ are positive functions. In some cases upper bounds for each eigenvalue are also estimated.

Assume that there exists an integrable function $F$ over $(0, a)$ such that

$$
\frac{a_{1}\left(x_{1}, y\right)}{a_{2}\left(x_{2}, y\right)} \leq \frac{F\left(x_{1}\right)}{F\left(x_{2}\right)}
$$

for any $x_{1}<x_{2}$ in $(0, a)$ and $1 / F$ is also integrable over $(0, a)$. Note that this assumption allows the coefficient $a_{1}$ to have jumps in both $x$ and $y$ directions and is easily satisfied, for example, if the coefficient $a_{1}$ is piecewise differentiable for $x$ over $(0, a)$. Under this assumption the application of the new results yields a $\gamma h^{-1}+o\left(h^{-1}\right)$ bound for the condition number of the preconditioned matrix and shows clearly how the coefficients $a_{1}$ and $a_{2}$ influence the constant $\gamma$, where $h$ is a meshsize in $x$-direction. This type of $O\left(h^{-1}\right)$ bounds was derived earlier only for a few limited cases, e.g. [11,18,21]. The assumption on the coefficients of partial differential equations in this paper is significantly weaker than previous ones. Also the new results give more accurate bounds even for the more restricted cases where the previous results are applicable.

The difference between considering all $\sigma_{i}$ and considering only $\sigma=\max _{i} \sigma_{i}$ for estimates of the condition number of block incomplete factorizations is essential. In practice, when a modified block incomplete factorization is applied to an elliptic equation most $\sigma_{i}$ are near 2 (see this paper), i.e., $\sigma_{i}=2+O(h)$, but some of them can be essentially larger than 2 if the coefficients of the elliptic equation have jumps or are not differentiable at some points [7,11,18,21]. In general, in such cases the old results $[7,11,18,21]$ can only provide an $O\left(h^{-2}\right)$ type upper bound for the condition number of the preconditioned matrix, i.e., a condition number of the same order as for the original difference matrix. However, results involving all $\sigma_{i}$ in the present paper still yield a $\gamma h^{-1}$ upper bound. The situation is the same if the coefficients oscillate frequently.

\section{Simple upper bounds for every eigenvalue}

Let $A$ be a symmetric matrix and $C=(X+L) X^{-1}\left(X+L^{\mathrm{T}}\right)$, where $X$ is a s.p.d. matrix and $L$ is a real matrix. We consider estimates of upper bounds for every eigenvalue of the matrix $C^{-1} A$ or, equivalently, for every generalized eigenvalue of $\lambda C \mathbf{x}=A \mathbf{x}$. To relate these two matrices we introduced a matrix of the form $\sigma X+L+L^{\mathrm{T}}$, see [7], where $\sigma$ is a constant. If $A \leq \sigma X+L+L^{\mathrm{T}}$ with $\sigma<2$, it was shown that $1 /(2-\sigma)$ is an upper bound of the maximum eigenvalue of the matrix $C^{-1} A$. In this section, we generalize this result to every eigenvalue. We start with the following basic result.

Theorem 1. Let $A$ be a symmetric matrix and $C=(X+L) X^{-1}\left(X+L^{\mathrm{T}}\right)$ with a s.p.d. matrix $X$. If there are two constants $\alpha_{i}$ and $\sigma_{i}$ such that $\alpha_{i} \leq \lambda_{i}\left(X^{-1} K\right) \leq \sigma_{i}$, where $K=A-L-L^{\mathrm{T}}$, then

$$
\lambda_{\min }\left(M\left(\alpha_{i}\right)\right) \leq \lambda_{i}\left(C^{-1} A\right) \leq \lambda_{\max }\left(M\left(\sigma_{i}\right)\right),
$$

where $M(\sigma)=(I+\widetilde{L})^{-1}+\left(I+\widetilde{L}^{\mathrm{T}}\right)^{-1}+(\sigma-2)(I+\widetilde{L})^{-1}\left(I+\widetilde{L}^{\mathrm{T}}\right)^{-1}$ and $\widetilde{L}=X^{-\frac{1}{2}} L X^{-\frac{1}{2}}$. 
Proof. Using first a similarity transformation and then a congruence transformation, we find that the equality $\lambda_{i}\left(X^{-1} K\right)=\lambda_{i}\left(X^{-\frac{1}{2}} K X^{-\frac{1}{2}}\right) \leq \sigma_{i}$ is equivalent to $\lambda_{i}\left(K-\sigma_{i} X\right) \leq 0$. Furthermore, by a direct calculation we find

$$
\begin{aligned}
\lambda_{i}\left(C^{-1} A\right)= & \lambda_{i}\left(X^{-\frac{1}{2}}\left(X+L^{\mathrm{T}}\right) C^{-1} A\left(X+L^{\mathrm{T}}\right)^{-1} X^{\frac{1}{2}}\right) \\
= & \lambda_{i}\left(X^{\frac{1}{2}}(X+L)^{-1} A\left(X+L^{\mathrm{T}}\right) X^{\frac{1}{2}}\right) \\
= & \lambda_{i}\left(X^{\frac{1}{2}}(X+L)^{-1}\left(K-\sigma_{i} X\right)\left(X+L^{\mathrm{T}}\right)^{-1} X^{\frac{1}{2}}\right. \\
& \left.+X^{\frac{1}{2}}(X+L)^{-1}\left(\left(\sigma_{i}-2\right) X+X+L+X+L^{\mathrm{T}}\right)\left(X+L^{\mathrm{T}}\right)^{-1} X^{\frac{1}{2}}\right) \\
= & \lambda_{i}\left(X^{\frac{1}{2}}(X+L)^{-1}\left(K-\sigma_{i} X\right)\left(X+L^{\mathrm{T}}\right)^{-1} X^{\frac{1}{2}}+M\left(\sigma_{i}\right)\right)
\end{aligned}
$$

Applying Weyl's theorem (c.f. Parlett [22], p. 192) shows that

$$
\begin{aligned}
\lambda_{i}\left(C^{-1} A\right) & =\lambda_{i}\left(X^{-\frac{1}{2}}\left(X+L^{\mathrm{T}}\right) C^{-1} A\left(X+L^{\mathrm{T}}\right)^{-1} X^{\frac{1}{2}}\right) \\
& \leq \lambda_{i}\left(X^{\frac{1}{2}}(X+L)^{-1}\left(K-\sigma_{i} X\right)\left(X+L^{\mathrm{T}}\right)^{-1} X^{\frac{1}{2}}\right)+\lambda_{\max }\left(M\left(\sigma_{i}\right)\right),
\end{aligned}
$$

which implies that $\lambda_{i}\left(C^{-1} A\right) \leq \lambda_{\max }\left(M\left(\sigma_{i}\right)\right)$ if $\lambda_{i}\left(X^{-1} K\right) \leq \sigma_{i}$. If $\alpha_{i} \leq$ $\lambda_{i}\left(X^{-1} K\right)$, then $\lambda_{\min }\left(M\left(\alpha_{i}\right)\right) \leq \lambda_{i}\left(C^{-1} A\right)$ follows in a similar way.

The following two corollaries give some situations in which Theorem 1 is applicable. The first one shows a simple upper bound for every eigenvalue of the preconditioned matrix $C^{-1} A$, which generalizes some previous results for the maximum eigenvalue in $[3,7]$.

Corollary 1. If the conditions of Theorem 1 hold and $\sigma_{i}<2$, then

$$
\lambda_{i}\left(C^{-1} A\right) \leq \lambda_{\max }\left(M\left(\sigma_{i}\right)\right) \leq \frac{1}{2-\sigma_{i}}
$$

In particular, if $\lambda_{i}\left(X^{-1} K\right)<2$, then $\lambda_{i}\left(C^{-1} A\right) \leq\left(2-\lambda_{i}\left(X^{-1} K\right)\right)^{-1}$.

Proof. The proof is essentially the same as that of Proposition 2.4 in [7].

Corollary 2. If the conditions of Theorem 1 hold, if $\kappa \geq \rho(M)$, where $M=$ $\left(I+\widetilde{L}^{\mathrm{T}}\right)^{-1}(I+\widetilde{L})^{-1}$, and if $\kappa^{-\frac{1}{2}}+\sigma_{i} \geq 2$, then

$$
\lambda_{i}\left(C^{-1} A\right) \leq\left(\sigma_{i}-2\right) \kappa+2 \kappa^{\frac{1}{2}} .
$$

Proof. The proof is essentially the same as that of Theorem 3.3 in [7].

If $\sigma=\sigma_{1}$, then Corollary 2 yields directly the result for the maximum eigenvalue of the matrix $C^{-1} A$ that was given in [7]. 


\section{Further estimates for the maximum eigenvalue}

As mentioned in the previous section $\lambda_{\max }\left(C^{-1} A\right) \leq(\sigma-2) \kappa+2 \kappa^{\frac{1}{2}}$ if $A \leq \sigma X+$ $L+L^{\mathrm{T}}$. However, as it turns out, $\sigma$ may be much larger than 2 if a modified block incomplete factorization method is used to solve the linear systems arising from elliptic equations discretized by a central difference scheme. Usually, $\kappa=O\left(h^{-2}\right)$ as we will see in the following section, where $h$ is a meshsize. If $\sigma-2>0$ is independent of $h$, which can occur if the coefficients of the elliptic equations have jumps in both $x$ and $y$ directions, see [11,18,21], inequality (4) gives only an $O\left(h^{-2}\right)$ upper bound for the maximum eigenvalue of the preconditioned matrix. To obtain a more accurate bound for the maximum eigenvalue we consider the problem in a general context in this section. Our aim is to derive an upper bound for the maximum eigenvalue for the matrix $C^{-1} A$ involving all $\sigma_{i}$. The approaches are applicable to estimates of upper bounds for each eigenvalue. To this end, we recall first the generalization of the well known inequality $\rho(A) \leq$ $\operatorname{tr}(A)$ for a symmetric positive semidefinite matrix $A$ given in [15] (see also [7]).

Lemma 1. Let $B=\left(B_{i j}\right)$ be an $m \times m$ block symmetric positive semidefinite matrix. Then

$$
\rho(B) \leq \sum_{i=1}^{m} \rho\left(B_{i i}\right)
$$

Theorem 2. Let $C=(X+L) X^{-1}\left(X+L^{\mathrm{T}}\right)^{-1}$ with a block diagonal s.p.d matrix $X=$ blockdiag $\left(X_{1}, X_{2}, \ldots, X_{m}\right)$ and let $A \leq G+L+L^{\mathrm{T}}$ be a symmetric matrix, where $G=\operatorname{blockdiag}\left(G_{1}, G_{2}, \ldots, G_{m}\right), X_{i}$ and $G_{i}$ are matrices of order $n_{i}$. Partition the matrix $M=\left(I+\widetilde{L}^{\mathrm{T}}\right)^{-1}(I+\widetilde{L})^{-1}$ into an $m \times m$ block matrix $M=\left(M_{i j}\right)_{m \times m}$, where $M_{i j}$ are $n_{i} \times n_{j}$ matrices and $\widetilde{L}=X^{-\frac{1}{2}} L X^{-\frac{1}{2}}$. Then

$$
\lambda_{\max }\left(C^{-1} A\right) \leq \min _{\mu<2}\left(\frac{1}{2-\mu}+\sum_{i \in S(\mu)}\left(\rho\left(X_{i}^{-1} G_{i}\right)-\mu\right) \rho\left(M_{i i}\right)\right),
$$

where $S(\mu)=\left\{i ; \rho\left(X_{i}^{-1} G_{i}\right)>\mu\right\}$.

Proof. Using the matrix $G$ instead of $\sigma_{i} X$ in the proof of Theorem 1 and following that proof we obtain that

$$
\begin{aligned}
\lambda_{i}\left(C^{-1} A\right) \leq & \lambda_{i}\left((I+\widetilde{L})^{-1}+\left(I+\widetilde{L}^{\mathrm{T}}\right)^{-1}+\right. \\
& \left.(I+\widetilde{L})^{-1}\left(X^{-\frac{1}{2}} G X^{-\frac{1}{2}}-2 I\right)\left(I+\widetilde{L}^{\mathrm{T}}\right)^{-1}\right) .
\end{aligned}
$$

Define a block diagonal matrix $R(\mu)=\operatorname{blockdiag}\left(R_{1}, R_{2}, \ldots, R_{m}\right)$ by

$$
R_{i}= \begin{cases}\left(\rho\left(X_{i}^{-1} G_{i}\right)-\mu\right) I_{n_{i}}, & \text { if } \rho\left(X_{i}^{-1} G_{i}\right)>\mu, \\ 0_{n_{i}}, & \text { otherwise, }\end{cases}
$$

where $I_{k}$ and $0_{k}$ denote the unit matrix and the zero matrix of order $k$, respectively. Applying (7) to the maximum eigenvalue of $C^{-1} A$ shows that 


$$
\begin{aligned}
\lambda_{\max }\left(C^{-1} A\right) & \leq \lambda_{\max }\left((I+\widetilde{L})^{-1}+\left(I+\widetilde{L}^{\mathrm{T}}\right)^{-1}\right. \\
& \left.+(\mu-2)(I+\widetilde{L})^{-1}\left(I+\widetilde{L}^{\mathrm{T}}\right)^{-1}+(I+\widetilde{L})^{-1} R(\mu)\left(I+\widetilde{L}^{\mathrm{T}}\right)^{-1}\right)
\end{aligned}
$$

which together with Weyl's theorem shows that

$$
\begin{aligned}
& \lambda_{\max }\left(C^{-1} A\right) \leq \lambda_{\max }\left((I+\widetilde{L})^{-1}+\left(I+\widetilde{L}^{\mathrm{T}}\right)^{-1}\right. \\
& \left.\quad+(\mu-2)(I+\widetilde{L})^{-1}\left(I+\widetilde{L}^{\mathrm{T}}\right)^{-1}\right)+\lambda_{\max }\left((I+\widetilde{L})^{-1} R(\mu)\left(I+\widetilde{L}^{\mathrm{T}}\right)^{-1}\right) .
\end{aligned}
$$

For any $\mu<2$, Corollary 1 yields the following bound for the first part on the right hand side of (8)

$$
\lambda_{\max }\left((I+\widetilde{L})^{-1}+\left(I+\widetilde{L}^{\mathrm{T}}\right)^{-1}+(\mu-2)(I+\widetilde{L})^{-1}\left(I+\widetilde{L}^{\mathrm{T}}\right)^{-1}\right) \leq \frac{1}{2-\mu},
$$

and applying Lemma 1 shows that

$$
\begin{aligned}
\lambda_{\max }\left((I+\widetilde{L})^{-1}\right. & \left.R(\mu)\left(I+\widetilde{L}^{\mathrm{T}}\right)^{-1}\right) \\
& =\lambda_{\max }\left(R(\mu)^{\frac{1}{2}}\left(I+\widetilde{L}^{\mathrm{T}}\right)^{-1}(I+\widetilde{L})^{-1} R(\mu)^{\frac{1}{2}}\right) \\
& \leq \sum_{i \in S(\mu)}\left(\rho\left(X_{i}^{-1} G_{i}\right)-\mu\right) \rho\left(M_{i i}\right) .
\end{aligned}
$$

Inequalities (8), (9) and (10) imply now the desired result.

Theorem 2 gives a new upper bound for the maximum eigenvalue of the preconditioned matrix $C^{-1} A$. As we will see in the next section this result presents an essential improvement of Corollary 1 and Corollary 2 in practice. The result can also be used to find a bound for every eigenvalue in the following way. Reorder $\rho\left(X_{i}^{-1} G_{i}\right)$ such that for $\kappa_{j}=\rho\left(X_{i(j)}^{-1} G_{i(j)}\right)$ it holds $\kappa_{1} \geq \kappa_{2} \geq \cdots \geq \kappa_{m}$, and for $\sum_{k=1}^{p-1} n_{k}<i \leq \sum_{k=1}^{p} n_{k}, p=1,2, \ldots, m$, define a block diagonal matrix $Q=\operatorname{blockdiag}\left(Q_{1}, Q_{2}, \ldots, Q_{m}\right)$ by

$$
Q_{k}= \begin{cases}X_{k}^{-\frac{1}{2}} G_{k} X_{k}^{-\frac{1}{2}}, & \text { if } \rho\left(X_{k}^{-1} G_{k}\right) \leq \kappa_{p}, \\ \kappa_{p} I_{n_{k}}, & \text { if } \rho\left(X_{k}^{-1} G_{k}\right)>\kappa_{p} .\end{cases}
$$

Write (7) in the following form

(11) $\lambda_{i}\left(C^{-1} A\right) \leq \lambda_{i}\left((I+\widetilde{L})^{-1}+\left(I+\widetilde{L}^{\mathrm{T}}\right)^{-1}+(I+\widetilde{L})^{-1}(Q-2 I)\left(I+\widetilde{L}^{\mathrm{T}}\right)^{-1}\right.$

$$
\left.(I+\widetilde{L})^{-1}\left(X^{-\frac{1}{2}} G X^{-\frac{1}{2}}-Q\right)\left(I+\widetilde{L}^{\mathrm{T}}\right)^{-1}\right) .
$$

It is straightforward to see that

$$
\lambda_{i}\left((I+\widetilde{L})^{-1}\left(X^{-\frac{1}{2}} G X^{-\frac{1}{2}}-Q\right)\left(I+\widetilde{L}^{\mathrm{T}}\right)^{-1}\right)=0 .
$$

Applying again Weyl's theorem to (11) yields

$$
\begin{aligned}
\lambda_{i}\left(C^{-1} A\right) \leq & \lambda_{\max }\left((I+\widetilde{L})^{-1}+\left(I+\widetilde{L}^{\mathrm{T}}\right)^{-1}+(I+\widetilde{L})^{-1}(Q-2 I)\left(I+\widetilde{L}^{\mathrm{T}}\right)^{-1}\right) \\
& +\lambda_{i}\left((I+\widetilde{L})^{-1}\left(X^{-\frac{1}{2}} G X^{-\frac{1}{2}}-Q\right)\left(I+\widetilde{L}^{\mathrm{T}}\right)^{-1}\right) \\
= & \lambda_{\max }\left((I+\widetilde{L})^{-1}+\left(I+\widetilde{L}^{\mathrm{T}}\right)^{-1}+(I+\widetilde{L})^{-1}(Q-2 I)\left(I+\widetilde{L}^{\mathrm{T}}\right)^{-1}\right)
\end{aligned}
$$


Therefore, Theorem 2 shows the following bound for the $i$ th eigenvalue of $C^{-1} A$,

$$
\lambda_{i}\left(C^{-1} A\right) \leq \min _{\mu<2, \mu \leq \kappa_{p}}\left(\begin{array}{c}
1 \\
2-\mu
\end{array}+\sum_{k \in S(\mu)}\left(\min \left(\kappa_{p}, \rho\left(X_{k}^{-1} G_{k}\right)\right)-\mu\right) \rho\left(M_{k k}\right)\right) .
$$

In practice, Theorem 2 frequently shows more accurate bounds for the maximum eigenvalue than the previous results. To make the estimate easier to apply we give the following corollary.

Corollary 3. If the conditions of Theorem 2 hold, then

$$
\lambda_{\max }\left(C^{-1} A\right) \leq 2\left(\sum_{i=1}^{m} \rho\left(M_{i i}\right)\right)^{\frac{1}{2}}+\sum_{i \in S(2)}\left(\rho\left(X_{i}^{-1} G_{i}\right)-2\right) \rho\left(M_{i i}\right) .
$$

Proof. Let $\rho_{i}=\rho\left(X_{i}^{-1} G_{i}\right)$ and define $r_{i}, i=1,2, \ldots, m$ by

$$
r_{i}= \begin{cases}2, & \text { if } \rho_{i} \leq 2 \\ \rho_{i}, & \text { if } \rho_{i}>2\end{cases}
$$

Using $r_{i}$ instead of $\rho\left(X_{i}^{-1} G_{i}\right)$ and applying Theorem 2 show that

$$
\begin{aligned}
\lambda_{\max }\left(C^{-1} A\right) & \leq \min _{\mu<2}\left(\frac{1}{2-\mu}+\sum_{i=1}^{m}\left(r_{i}-\mu\right) \rho\left(M_{i i}\right)\right) \\
\leq & 2\left(\sum_{i=1}^{m} \rho\left(M_{i i}\right)\right)^{\frac{1}{2}}+\sum_{i \in S(2)}\left(\rho_{i}-2\right) \rho\left(M_{i i}\right)
\end{aligned}
$$

The minimum is taken for $\mu=2-\left(\sum_{i=1}^{m} \rho\left(M_{i i}\right)\right)^{-\frac{1}{2}}$.

If $A \leq \sigma X+L+L^{\mathrm{T}}$ is symmetric positive semidefinite with $1 \leq \sigma \leq 2$, where $X$ is, in addition, a block diagonal matrix and $L$ is a strictly lower block triangular matrix, we showed in [7] that $2(\sigma-1) m+2-\sigma(\leq 2 m)$ is another upper bound for the maximum eigenvalue of the matrix $C^{-1} A$. Bounds which do not depend on the coefficients of the matrix but only on the number of blocks in its block matrix partitioning can be very useful in many cases. Under the strong assumption that $A$ is a Stieltjes matrix and other additional conditions which are stronger than $\sigma<2$, this bound can be reduced to $m+1$ (see [18]). We now improve this type of upper bound. Another upper bound involving all $\sigma_{i}$ is derived for the maximum eigenvalue of the preconditioned matrix $C^{-1} A$. The new result allows $\sigma_{i}>2$. If the result is applied to a symmetric matrix $A$ such that $0 \leq A \leq \sigma X+L+L^{\mathrm{T}}$ with $\sigma \leq 2$, we obtain an upper bound even smaller than our previous bound $2(\sigma-1) m+2-\sigma$. In particular, if $C \leq A$, we have $\lambda_{\max }\left(C^{-1} A\right) \leq m+1$. In the rest of this section we assume that $L$ is a strictly lower block triangular matrix. 
Theorem 3. Let $A \leq G+L+L^{\mathrm{T}}$ be a symmetric positive semidefinite matrix, where $G=\operatorname{blockdiag}\left(G_{1}, G_{2}, \ldots, G_{m}\right)$, and $C=(X+L) X^{-1}\left(X+L^{\mathrm{T}}\right)$ with a s.p.d matrix $X=\operatorname{blockdiag}\left(X_{1}, X_{2}, \ldots, X_{m}\right)$, where $G_{i}$ and $X_{i}$ are square matrices of order $n_{i}$. Partition $\left(I+X^{-\frac{1}{2}} L X^{-\frac{1}{2}}\right)=\left(T_{i j}\right)_{m \times m}$ into an $m \times m$ block matrix consistently with the partitioning of $G$. If $\beta C \leq A$, where $\beta$ is a nonnegative constant, then

(14) $\lambda_{\max }\left(C^{-1} A\right) \leq \sum_{\rho_{i} \leq 2} \rho_{i}+\sum_{\rho_{i}>2}\left(2+\left(\rho_{i}-2\right) \sum_{k=i}^{m} \rho\left(T_{k i} T_{k i}^{\mathrm{T}}\right)\right)-\beta(m-1)$,

where $\rho_{i}=\rho\left(X_{i}^{-1} G_{i}\right)$.

Proof. Define two block diagonal matrices

$$
\begin{gathered}
P=\operatorname{blockdiag}\left(\rho_{1} I_{n_{1}}, \rho_{2} I_{n_{2}}, \ldots, \rho_{m} I_{n_{m}}\right), \\
R=\operatorname{blockdiag}\left(r_{1} I_{n_{1}}, r_{2} I_{n_{2}}, \ldots, r_{m} I_{n_{m}}\right),
\end{gathered}
$$

where $r_{i}$ is defined by (13). It follows from (7) that

$$
\begin{gathered}
\lambda_{i}\left(C^{-1} A\right) \leq \lambda_{i}\left((I+\widetilde{L})^{-1}+\left(I+\widetilde{L}^{\mathrm{T}}\right)^{-1}+(I+\widetilde{L})^{-1}(P-2 I)\left(I+\widetilde{L}^{\mathrm{T}}\right)^{-1}\right) \\
\leq \lambda_{i}\left((I+\widetilde{L})^{-1}+\left(I+\widetilde{L}^{\mathrm{T}}\right)^{-1}+(I+\widetilde{L})^{-1}(P-R)\left(I+\widetilde{L}^{\mathrm{T}}\right)^{-1}\right. \\
\left.+(I+\widetilde{L})^{-1}(R-2 I)\left(I+\widetilde{L}^{\mathrm{T}}\right)^{-1}\right)
\end{gathered}
$$

On the other hand, for any matrix $D$ a simple computation shows that

$$
\begin{aligned}
& (I+\widetilde{L})^{-1} D\left(I+\widetilde{L}^{\mathrm{T}}\right)^{-1} \\
= & \left(I-(I+\widetilde{L})^{-1} \widetilde{L}\right) D\left(I-\widetilde{L}^{\mathrm{T}}\left(I+\widetilde{L}^{\mathrm{T}}\right)^{-1}\right) \\
= & D-(I+\widetilde{L})^{-1} \widetilde{L} D-D \widetilde{L}^{\mathrm{T}}\left(I+\widetilde{L}^{\mathrm{T}}\right)^{-1}+(I+\widetilde{L})^{-1} \widetilde{L} D \widetilde{L}^{\mathrm{T}}\left(I+\widetilde{L}^{\mathrm{T}}\right)^{-1} \\
= & D-(I+\widetilde{L})^{-1}(I+\widetilde{L}-I) D-D\left(I+\widetilde{L}^{\mathrm{T}}-I\right)\left(I+\widetilde{L}^{\mathrm{T}}\right)^{-1} \\
& +(I+\widetilde{L})^{-1} \widetilde{L} D \widetilde{L}^{\mathrm{T}}\left(I+\widetilde{L}^{\mathrm{T}}\right)^{-1} \\
= & (I+\widetilde{L})^{-1} D+D\left(I+\widetilde{L}^{\mathrm{T}}\right)^{-1}-D+(I+\widetilde{L})^{-1} \widetilde{L} D \widetilde{L}^{\mathrm{T}}\left(I+\widetilde{L}^{\mathrm{T}}\right)^{-1} .
\end{aligned}
$$

Therefore, for the $i$ th eigenvalue of the matrix $C^{-1} A$ inequality (15) becomes

$$
\begin{gathered}
\lambda_{i}\left(C^{-1} A\right) \leq \lambda_{i}\left((I+\widetilde{L})^{-1}(P-R+I)+(P-R+I)\left(I+\widetilde{L}^{\mathrm{T}}\right)^{-1}+R-P\right. \\
\left.+(I+\widetilde{L})^{-1} \widetilde{L}(P-R) \widetilde{L}^{\mathrm{T}}\left(I+\widetilde{L}^{\mathrm{T}}\right)^{-1}+(I+\widetilde{L})^{-1}(R-2 I)\left(I+\widetilde{L}^{\mathrm{T}}\right)^{-1}\right)
\end{gathered}
$$

Since the matrix $(I+\widetilde{L})^{-1} \widetilde{L}(P-R) \widetilde{L}^{\mathrm{T}}\left(I+\widetilde{L}^{\mathrm{T}}\right)^{-1}$ is negative semidefinite, applying Weyl's theorem to (16) we have

$$
\begin{gathered}
\lambda_{i}\left(C^{-1} A\right) \leq \lambda_{i}\left((I+\widetilde{L})^{-1}(P-R+I)+(P-R+I)\left(I+\widetilde{L}^{\mathrm{T}}\right)^{-1}\right. \\
\left.+R-P+(I+\widetilde{L})^{-1}(R-2 I)\left(I+\widetilde{L}^{\mathrm{T}}\right)^{-1}\right) .
\end{gathered}
$$

In particular, assumption $\beta C \leq A$ implies

$$
\begin{gathered}
\beta \leq \lambda_{\min }\left(C^{-1} A\right) \leq \lambda_{\min }\left((I+\widetilde{L})^{-1}(P-R+I)+(P-R+I)\left(I+\widetilde{L}^{\mathrm{T}}\right)^{-1}\right. \\
\left.+R-P+(I+\widetilde{L})^{-1}(R-2 I)\left(I+\widetilde{L}^{\mathrm{T}}\right)^{-1}\right),
\end{gathered}
$$


which shows that the matrix

$$
\begin{aligned}
& (I+\widetilde{L})^{-1}(P-R+I)+(P-R+I)\left(I+\widetilde{L}^{\mathrm{T}}\right)^{-1} \\
& +R-P-\beta I+(I+\widetilde{L})^{-1}(R-2 I)\left(I+\widetilde{L}^{\mathrm{T}}\right)^{-1}
\end{aligned}
$$

is positive semidefinite. Applying (17) to the maximum eigenvalue yields

$$
\begin{gathered}
\lambda_{\max }\left(C^{-1} A\right) \leq \lambda_{\max }\left((I+\widetilde{L})^{-1}(P-R+I)+(P-R+I)\left(I+\widetilde{L}^{\mathrm{T}}\right)^{-1}\right. \\
\left.+R-P-\beta I+(I+\widetilde{L})^{-1}(R-2 I)\left(I+\widetilde{L}^{\mathrm{T}}\right)^{-1}\right)+\beta .
\end{gathered}
$$

Furthermore, it is straightforward to show that the $(k, k)$ block of $(I+\widetilde{L})^{-1}(R-$ $2 I)\left(I+\widetilde{L}^{\mathrm{T}}\right)^{-1}$ is given by

$$
\sum_{i=1}^{k}\left(r_{i}-2\right) T_{k i} T_{k i}^{\mathrm{T}}
$$

Hence, applying Lemma 1 to (18) shows that

$$
\begin{aligned}
\lambda_{\max } & \left(C^{-1} A\right) \leq \sum_{i=1}^{m}\left(\rho_{i}-r_{i}\right)+2 m-\beta m+\sum_{k=1}^{m} \sum_{i=1}^{k}\left(r_{i}-2\right) \rho\left(T_{k i} T_{k i}^{\mathrm{T}}\right)+\beta \\
= & \sum_{\rho_{i} \leq 2} \rho_{i}+\sum_{\rho_{i}>2} 2+\sum_{i=1}^{m}\left(r_{i}-2\right) \sum_{k=i}^{m} \rho\left(T_{k i} T_{k i}^{\mathrm{T}}\right)-(m-1) \beta \\
= & \sum_{\rho_{i} \leq 2} \rho_{i}+\sum_{\rho_{i}>2}\left(\left(\rho_{i}-2\right) \sum_{k=i}^{m} \rho\left(T_{k i} T_{k i}^{\mathrm{T}}\right)+2\right)-(m-1) \beta,
\end{aligned}
$$

which completes the proof.

If $\rho_{i} \leq 2, i=1,2, \ldots, m$, Theorem 3 yields immediately the following result.

Corollary 4. If the conditions of Theorem 3 hold with $\rho_{i} \leq 2$, then

$$
\lambda_{\max }\left(C^{-1} A\right) \leq \sum_{i=1}^{m} \rho_{i}-\beta(m-1) .
$$

In particular, if $C \leq A$ then $\lambda_{\max }\left(C^{-1} A\right) \leq m+1$.

\section{Application to elliptic equations}

As an application of the results of the previous sections, we consider an elliptic equation in two dimensions

$$
\begin{gathered}
-\frac{\partial}{\partial x}\left(a_{1} \frac{\partial}{\partial x} u\right)-\frac{\partial}{\partial y}\left(a_{2} \frac{\partial}{\partial y} u\right)=f \quad \text { on } \Omega, \\
u=g \text { on } \Gamma_{0} \\
\partial u=k \text { on } \Gamma_{1}=\partial \Omega \backslash \Gamma_{0},
\end{gathered}
$$

where $\Omega=(0, a) \times(0, b)$, the coefficients $a_{1}$ and $a_{2}$ are positive functions. We consider the following three kinds of typical boundary conditions 
- Problem 1: $\Gamma_{0}=\partial \Omega$,

- Problem 2: $\Gamma_{1}=\{(x, y), 0<x<a, y=0\}$,

- Problem 3: $\Gamma_{0}=\{(x, y), 0<y<b, x=a\}$,

which serve well to illustrate the bounds in the previous sections. For other kinds of boundary conditions the estimates are similar. Discretizing the equation by a central difference scheme with a grid of meshsize $h$ in $x$ direction and $\delta$ in $y$ direction and ordering the mesh columnwise yields the following linear system

$$
A \mathbf{x}=\mathbf{b},
$$

where $A$ is an $m \times m$ block tridiagonal matrix of the form

$$
A=\operatorname{blocktridiag}\left(A_{i, i-1}, A_{i i}, A_{i, i+1}\right)
$$

and $A_{i i}$ is a tridiagonal matrix of order $n$.

We compute a modified block incomplete factorization preconditioner $C=$ $(D-L) D^{-1}\left(D-L^{\mathrm{T}}\right)$ for $A$ as follows:

$$
\begin{aligned}
& D_{1}=A_{11}, \\
& D_{i}=A_{i i}-A_{i, i-1} X_{i-1} A_{i-1, i}+D_{i}^{\prime}, \quad i=2, \ldots, m,
\end{aligned}
$$

where $X_{i}, i \geq 1$, is a sparse approximation to $D_{i}^{-1}$ such that the off-diagonal entries of $X_{i}$ are not larger than $D_{i}^{-1}$, and $D_{i}^{\prime}$ is a diagonal matrix determined by

$$
D_{i}^{\prime} \mathbf{e}=A_{i, i-1}\left(X_{i-1}-D_{i-1}^{-1}\right) A_{i-1, i} \mathbf{e},
$$

where $\mathbf{e}=(1,1, \ldots, 1)^{\mathrm{T}}$. Hence, we have

$$
\begin{aligned}
& D_{1} \mathbf{e}=A_{11} \mathbf{e}, \\
& D_{i} \mathbf{e}=\left(A_{i i}-A_{i, i-1} D_{i-1}^{-1} A_{i-1, i}\right) \mathbf{e} .
\end{aligned}
$$

It is straightforward to show that $A-C$ is a $Z$-matrix, i.e., all off diagonal entries are nonpositive, and $(A-C) \mathbf{e}=0$. This implies that for the lower eigenvalue bound we have $\lambda_{\min }\left(C^{-1} A\right) \geq 1$. Therefore, all upper bounds of the maximum eigenvalue of $C^{-1} A$ given in this section are bounds of the condition number of the preconditioned matrix $C^{-1} A$. Note that for this application for the corresponding matrix $\widetilde{L}$, it holds $\widetilde{L}=-D^{-\frac{1}{2}} L D^{-\frac{1}{2}}$, where $D=\operatorname{blockdiag}\left(D_{1}, D_{2}, \ldots, D_{m}\right)$.

\subsection{General analysis}

The task in this subsection is to derive an upper bound for the maximum eigenvalue of the preconditioned matrix $C^{-1} A$ for problems 1,2 and 3 . Let $\triangle_{i}=-A_{i, i+1}$. Then $A_{i i} \mathbf{e} \geq\left(\triangle_{i}+\triangle_{i-1}\right) \mathbf{e}, i \geq 1$ for problems 1 and 2 and $A_{11} \mathbf{e} \geq \triangle_{1} \mathbf{e}, A_{i i} \mathbf{e} \geq\left(\triangle_{i}+\triangle_{i-1}\right) \mathbf{e}, i \geq 2$ for problem 3. This implies that $D_{1} \mathbf{e} \geq \triangle_{1} \mathbf{e}$. By induction and using (21) it is readily shown that

$$
D_{i} \mathbf{e}=\left(A_{i i}-\triangle_{i-1} D_{i-1}^{-1} \triangle_{i-1}\right) \mathbf{e} \geq \triangle_{i} \mathbf{e} .
$$


Denote $\beta_{1}=0$ and

$$
\beta_{i}=\max \left(\frac{a_{1}\left(\left(i-i_{0}-1 / 2\right) h,\left(1-k_{0}\right) \delta\right)}{a_{1}\left(\left(i-i_{0}+1 / 2\right) h,\left(1-k_{0}\right) \delta\right)}, \ldots, \frac{a_{1}\left(\left(i-i_{0}-1 / 2\right) h,\left(n-k_{0}\right) \delta\right)}{a_{1}\left(\left(i-i_{0}+1 / 2\right) h,\left(n-k_{0}\right) \delta\right)}\right)
$$

for $i \geq 2$, where $i_{0}=0$ for problems 1 and $2, i_{0}=1$ for problem $3, k_{0}=0$ for problem 1 and $k_{0}=1$ for problems 2 and 3 . A simple computation shows that for $i \geq 2$

$$
\begin{array}{r}
\left(1+\beta_{i}\right) D_{i} \mathbf{e}=A_{i i} \mathbf{e}+\left(\beta_{i} A_{i i}-\left(1+\beta_{i}\right) A_{i, i-1} D_{i-1}^{-1} A_{i-1, i}\right) \mathbf{e} \\
\geq A_{i i} \mathbf{e}+\left(\beta_{i}\left(\triangle_{i}+\triangle_{i-1}\right)-\left(1+\beta_{i}\right) \triangle_{i}\right) \mathbf{e} \geq A_{i i} \mathbf{e} .
\end{array}
$$

In addition, $\left(1+\beta_{i}\right) D_{i}-A_{i i}=\beta_{i} D_{i}+\left(D_{i}-A_{i i}\right)$ is a $Z$-matrix. Therefore, $(1+$ $\left.\beta_{i}\right) D_{i}-A_{i i} \geq 0$ and

$$
\rho\left(D_{i}^{-1} A_{i i}\right) \leq 1+\beta_{i}, \quad i=1,2, \ldots, m .
$$

If $a_{1}(x, y)$ is non-decreasing for $x$ then $\beta_{i} \leq 1$, and an application of Theorem 3 shows that

$$
\lambda_{\max }\left(C^{-1} A\right) \leq \sum_{i=1}^{m} \beta_{i}+1 \leq m
$$

because $\beta_{1}=0$ and $C \leq A$. If the coefficient $a_{1}$ is non-increasing for the first variable $x$, for problems 1 and 2 we reorder the mesh in $x$ direction from right to left or first do a transformation $x \mapsto a-x$. Inequality (24) gives still an upper bound for the maximum eigenvalue of the corresponding preconditioned matrix.

To further analyze our ILU preconditioner we assume that there exists an integrable function $F$ over $(0, a)$ such that

$$
\frac{a_{1}\left(x_{1}, y\right)}{a_{1}\left(x_{2}, y\right)} \leq \frac{F\left(x_{1}\right)}{F\left(x_{2}\right)}
$$

for any $x_{1} \leq x_{2}$ in $(0, a)$ and $1 / F$ is also integrable over $(0, a)$.

Indeed this condition is not strict. The assumption allows the coefficient $a_{1}$ to have some jumps in both $x$ and $y$ directions. For example, assume that $a_{1}$ is a piecewise differentiable function for the first variable $x$ over $\Omega$, i.e., there exist $0=a_{1}<a_{2}<\cdots<a_{k+1}=a$ such that $a_{1}$ is differentiable over $\left(a_{i}, a_{i+1}\right)$ for $i=1,2, \ldots, k$. Let

$$
g_{i}(x)=\max _{0 \leq y \leq b}-\frac{\partial a_{1}(x, y)}{\partial x} / a_{1}(x, y),
$$

for $x \in\left(a_{i}, a_{i+1}\right), i=1, \ldots, k$. If the function $g_{i}$ is integrable over $\left(a_{i}, a_{i+1}\right)$ we show now that there is a function $F$ such that (25) holds.

Lemma 2. Let $\theta: \Omega \mapsto \mathbb{R}$ and $m_{1}, m_{2}:(0, a) \mapsto \mathbb{R}$ be integrable functions. If there exist $\eta$ and $\xi\left(x_{1} \leq \eta, \xi \leq x_{2}\right)$ such that

$$
m_{1}(\eta)\left(x_{2}-x_{1}\right) \leq \theta\left(x_{2}, y\right)-\theta\left(x_{1}, y\right) \leq m_{2}(\xi)\left(x_{2}-x_{1}\right)
$$

uniformly in $y$ for any $x_{1} \leq x_{2}$ in $\Omega$, then

$$
\int_{x_{1}}^{x_{2}} m_{1}(x) d x \leq \theta\left(x_{2}, y\right)-\theta\left(x_{1}, y\right) \leq \int_{x_{1}}^{x_{2}} m_{2}(x) d x .
$$


Proof. Under the assumptions of the lemma, we have for any $x_{1}=w_{1}<w_{2}<$ $\cdots<w_{p}=x_{2}$ that

$$
\theta\left(x_{2}, y\right)-\theta\left(x_{1}, y\right)=\sum_{1}^{p}\left(\theta\left(w_{i+1}, y\right)-\theta\left(w_{i}, y\right)\right) \leq \sum_{1}^{p} m_{2}\left(\xi_{i}\right)\left(w_{i+1}-w_{i}\right),
$$

where $w_{i} \leq \xi_{i} \leq w_{i+1}$. Setting $p \rightarrow \infty$ and letting $w_{i+1}-w_{i} \rightarrow 0$ we obtain the second inequality. The first one follows in a similar way.

For $x \in(0, a)$ define $k(x)=\max \left\{i: x>a_{i}\right\}, r_{1}=1$, and

$$
\begin{aligned}
& r_{i}=\max _{0 \leq y \leq b} \frac{a_{1}\left(a_{i}-0, y\right)}{a_{1}\left(a_{i}+0, y\right)}, \quad i=2, \ldots, k, \\
& g(x)= \begin{cases}g_{i}(x), & \text { if } x \in\left(a_{i}, a_{i+1}\right), \\
0, & \text { if } x=a_{i} .\end{cases}
\end{aligned}
$$

Here $g$ is clearly an integrable function over $(0, a)$. For any $x_{1}<x_{2}$ in $(0, a)$, if $k\left(x_{1}\right) \neq k\left(x_{2}\right)$ we write

$$
\begin{gathered}
\frac{a_{1}\left(x_{1}, y\right)=}{a_{1}\left(x_{2}, y\right)}=\frac{a_{1}\left(x_{1}, y\right)}{a_{1}\left(a_{k\left(x_{1}\right)+1}-0, y\right)} \cdots \frac{a_{1}\left(a_{k\left(x_{2}\right)-1}+0, y\right)}{a_{1}\left(a_{k\left(x_{2}\right)}-0, y\right)} \frac{a_{1}\left(a_{k\left(x_{2}\right)}+0, y\right)}{a_{1}\left(x_{2}, y\right)} \\
\prod_{k=k\left(x_{1}\right)+1}^{k\left(x_{2}\right)} a_{1}\left(a_{k}-0, y\right) .
\end{gathered}
$$

Since $a_{1}$ is differentiable over $\left(a_{i}, a_{i+1}\right)$ and $a_{1}(x, y)$ is positive, by definition of $g$ there exists a $\xi\left(z_{1}<\xi<z_{2}\right)$ such that

$$
\log a_{1}\left(z_{1}, y\right)-\log a_{1}\left(z_{2}, y\right) \leq g(\xi)\left(z_{2}-z_{1}\right)
$$

for any $z_{1}<z_{2}$ in $\left(a_{i}, a_{i+1}\right)$. Applying Lemma 2 shows that

$$
\log a_{1}\left(z_{1}, y\right)-\log a_{1}\left(z_{2}, y\right) \leq \int_{z_{1}}^{z_{2}} g(x) d x .
$$

Therefore, using the above and (26) shows that the following inequality holds,

$$
\frac{a_{1}\left(x_{1}, y\right)}{a_{1}\left(x_{2}, y\right)} \leq r_{k\left(x_{1}\right)+1} \cdots r_{k\left(x_{2}\right)} \exp \left(\int_{x_{1}}^{x_{2}} g(x) d x\right)=\frac{F\left(x_{1}\right)}{F\left(x_{2}\right)},
$$

where $F(x)=r_{1}^{-1} \cdots r_{k(x)}^{-1} \exp \left(-\int_{0}^{x} g(x) d x\right)$. It is easily seen that (27) holds for any $x_{1}<x_{2}$ in $(0, a)$ if $k\left(x_{1}\right)=k\left(x_{2}\right)$.

Now we continue our analysis for modified block incomplete factorizations for elliptic equations. Inequality (22) implies that $D_{i}^{-1} \triangle_{i} \mathbf{e} \leq \mathbf{e}$. Hence,

$$
\begin{aligned}
D_{i}^{-1} \triangle_{i-1} \mathbf{e} & \leq D_{i}^{-1} \triangle_{i} \triangle_{i-1} \triangle_{i}^{-1} \mathbf{e} \\
& \leq D_{i}^{-1} \triangle_{i} \frac{F\left(\left(i-i_{0}-1 / 2\right) h\right)}{F\left(\left(i-i_{0}+1 / 2\right) h\right)} \mathbf{e} \leq \frac{F\left(\left(i-i_{0}-1 / 2\right) h\right)}{F\left(\left(i-i_{0}+1 / 2\right) h\right)} \mathbf{e}
\end{aligned}
$$


Write the lower block tridiagonal matrix $(I+\widetilde{L})^{-1}$ in block form

$$
(I+\widetilde{L})^{-1}=\left(T_{i j}\right)=\sum_{r=0}^{m-1}\left(D^{-\frac{1}{2}} L D^{-\frac{1}{2}}\right)^{r},
$$

where $T_{i i}=I, T_{i j}=D_{i}^{-\frac{1}{2}} \triangle_{i-1} D_{i-1}^{-1} \cdots D_{j+1}^{-1} \triangle_{j} D_{j}^{-\frac{1}{2}}$ for $i>j$ and $T_{i j}=0$ for $i<j$. Partition $M=\left(I+\widetilde{L}^{\mathrm{T}}\right)^{-1}(I+\widetilde{L})^{-1}$ into an $m \times m$ block matrix $\left(M_{i j}\right)$ consistently with the partitioning of $A$. Then for any $k>i$, we have

$$
\begin{aligned}
D_{i}^{-1 / 2} & T_{k i}^{\mathrm{T}} T_{k i} D_{i}^{1 / 2} \mathbf{e} \\
& =\left(D_{i}^{-1} \triangle_{i}\right) \cdots\left(D_{k-1}^{-1} \triangle_{k-1}\right)\left(D_{k}^{-1} \triangle_{k-1}\right) \cdots\left(D_{i+1}^{-1} \triangle_{i}\right) \mathbf{e} \\
& =\frac{F\left(\left(i-i_{0}+1 / 2\right) h\right)}{F\left(\left(k-i_{0}+1 / 2\right) h\right)} \mathbf{e},
\end{aligned}
$$

which implies that the spectral radius

$$
\rho\left(T_{i k} T_{i k}^{\mathrm{T}}\right)=\rho\left(D_{i}^{-\frac{1}{2}} T_{i k} T_{i k}^{\mathrm{T}} D_{i}^{\frac{1}{2}}\right) \leq \frac{F\left(\left(i-i_{0}+1 / 2\right) h\right)}{F\left(\left(k-i_{0}+1 / 2\right) h\right)},
$$

because $D_{i}^{-\frac{1}{2}} T_{i k} T_{i k}^{\mathrm{T}} D_{i}^{\frac{1}{2}}$ is nonnegative. Therefore,

$$
\rho\left(M_{i i}\right)=\rho\left(\sum_{k=i}^{m} T_{k i}^{\mathrm{T}} T_{k i}\right) \leq \sum_{k=i}^{m} \rho\left(T_{k i}^{\mathrm{T}} T_{k i}\right) \leq \sum_{k=i}^{m} F\left(\left(k-i_{0}+1 / 2\right) h\right) .
$$

Let $S=\left\{i: F\left(\left(i-i_{0}-1 / 2\right) h\right)>F\left(\left(i-i_{0}+1 / 2\right) h\right)\right\}$. Since the spectral radius satisfies $\rho\left(D_{i}^{-1} A_{i i}\right) \leq 1+F\left(\left(i-i_{0}-1 / 2\right) h\right) / F\left(\left(i-i_{0}+1 / 2\right) h\right)$, to derive an upper bound for the maximum eigenvalue of the preconditioned matrix by Corollary 3 we need to estimate $\sum_{i=1}^{m} \rho\left(M_{i i}\right)$ and $\sum_{i \in S}\left(F\left(\left(i-i_{0}-1 / 2\right) h\right) / F((i-\right.$ $\left.\left.\left.i_{0}+1 / 2\right) h\right)-1\right) \rho\left(M_{i i}\right)$. By definition of integration it follows from (28) that

$$
\begin{aligned}
& \sum_{i=1}^{m} \rho\left(M_{i i}\right) \leq \sum_{i=1}^{m} \sum_{k=i}^{m} \frac{F\left(\left(i-i_{0}+1 / 2\right) h\right)}{F\left(\left(k-i_{0}+1 / 2\right) h\right)} \approx h^{-2} \int_{0}^{a} \int_{x}^{a} \frac{F(x)}{F(y)} d y d x . \\
& \sum_{i \in S}\left(\begin{array}{l}
F\left(\left(i-i_{0}-1 / 2\right) h\right) \\
F\left(\left(i-i_{0}+1 / 2\right) h\right)
\end{array}-1\right) \rho\left(M_{i i}\right) \\
& =\sum_{i \in S} \sum_{k=i}^{m} \frac{F\left(\left(i-i_{0}-1 / 2\right) h\right)-F\left(\left(i-i_{0}+1 / 2\right) h\right)}{\left.F\left(k-i_{0}+1 / 2\right) h\right)} .
\end{aligned}
$$

For $x \in(0, a)$ let $E_{x} \subset(0, a)$ denote the maximum subinterval including $x$ such that $F(y) \geq F(z)$ for any $y<z$ in $E_{x}$ if such an interval exists for $x$. Note that we do not refer to a single point as an interval. It is straightforward that if $y \in E_{x}$ then $E_{y}=E_{x}$. Therefore, $E_{x}$ is the maximum connected component in the sense that $F(y) \geq F(z)$ for any $y<z$ in $E_{x}$. Let $U$ be the set of all these maximum connected components over $(0, a)$. We find that 


$$
\begin{aligned}
\sum_{i \in S} \sum_{k=i}^{m} \frac{F\left(\left(i-i_{0}-1 / 2\right) h\right)-F\left(\left(i-i_{0}+1 / 2\right) h\right)}{F\left(\left(k-i_{0}+1 / 2\right) h\right)} \\
\quad \approx \sum_{E \in U} \sum_{i h \in E} \sum_{k=i}^{m} \frac{F\left(\left(i-i_{0}-1 / 2\right) h\right)-F\left(\left(i-i_{0}+1 / 2\right) h\right)}{F\left(\left(k-i_{0}+1 / 2\right) h\right)} .
\end{aligned}
$$

For an interval $E$ denote the left and the right end points of $E$ by $l_{E}$ and $r_{E}$, respectively. Let $p$ and $q$ be the minimum and the maximum integers satisfying $\left(p-i_{0}\right) h \in E$ and $\left(q-i_{0}\right) h \in E$. Then

$$
\begin{aligned}
& \sum_{i h \in E} \sum_{k=i}^{m} \frac{F\left(\left(i-i_{0}-1 / 2\right) h\right)-F\left(\left(i-i_{0}+1 / 2\right) h\right)}{F\left(\left(k-i_{0}+1 / 2\right) h\right)} \\
& =\sum_{i=p}^{q} \sum_{k=i}^{m} \frac{F\left(\left(i-i_{0}-1 / 2\right) h\right)-F\left(\left(i-i_{0}+1 / 2\right) h\right)}{F\left(\left(k-i_{0}+1 / 2\right) h\right)} \\
& =\sum_{k=p}^{m} F\left(\left(k-i_{0}+1 / 2\right) h\right) \sum_{i=p}^{\min (k, q)}\left(F\left(\left(i-i_{0}-1 / 2\right) h\right)-F\left(\left(i-i_{0}+1 / 2\right) h\right)\right) \\
& \leq \sum_{k=p}^{m} F\left(l_{E}+0\right)-F\left(\min \left(\left(k-i_{0}+1 / 2\right) h, r_{E}-0\right)\right) \\
& \approx h^{-1} \int_{l_{E}}^{a} F\left(\left(k-i_{0}+1 / 2\right) h\right)
\end{aligned}
$$

Therefore, if $\sum_{E \in U} \int_{l_{E}}^{a} \frac{F\left(l_{E}+0\right)-F\left(\min \left(y, r_{E}-0\right)\right)}{F(y)} d y$ exists, applying Corollary 3 we have the following upper bound:

(30) $\gamma=2\left(\int_{0}^{a} \int_{x}^{a} \frac{F(x)}{F(y)} d y d x\right)^{\frac{1}{2}}+\sum_{E \in U} \int_{l_{E}}^{a} \frac{F\left(l_{E}+0\right)-F\left(\min \left(y, r_{E}-0\right)\right)}{F(y)} d y$.

There is no previous result to estimate the constant of $h^{-1}$ for $O\left(h^{-1}\right)$ type upper bounds of the maximum eigenvalue except in a few limit cases $[7,11,18$, 21]. The bound (29) shows clearly how the coefficient $a_{1}$ of the elliptic equation influences the upper bound of the condition number of the preconditioned matrix.

If $a_{1}(x, y)=\chi(x) \varphi(y)$, where $\chi$ and $\varphi$ are functions defined on $(0, a)$ and $(0, b)$, respectively, we can simply choose $F(x)=\chi(x)$. In general, it is not hard to give a rough estimate for the constant $\gamma$. For example, assume that $a_{1}$ is differentiable for $x$ in $\Omega$ and

$$
\max _{(x, y) \in \Omega}-\frac{\partial a_{1}(x, y)}{\partial x} / a_{1}(x, y) \leq M .
$$

If $M \leq 0$ than $a_{1}(x, y)$ is non-decreasing for $x$. Inequality (24) shows a simple upper bound $m$. If $M \geq 0$ it follows from our analysis that we can choose $F(x)=\exp (-M x)$. An elementary computation shows that 


$$
\gamma \leq \frac{1}{M}\left(\mathrm{e}^{M a}-M a-1+2\left(\mathrm{e}^{M a}-M a-1\right)^{1 / 2}\right) .
$$

If $a_{1}$ is piecewise differentiable for $x$ in $\Omega$, as it turns out, one can also derive a somewhat complex upper bound for $\gamma$ by assuming that $g_{i}(x) \leq M_{i}$ for $x \in$ $\left(a_{i}, a_{i+1}\right)$.

The upper bound $\gamma h^{-1}+o\left(h^{-1}\right)$ in this subsection covers all previous results of $O\left(h^{-1}\right)$ type upper bounds for the maximum eigenvalue, see [7,11, 18,21], and is applicable to elliptic equations if the condition (25) is satisfied, which is weaker than that the coefficient $a_{1}$ is piecewise differentiable for $x$ in $\Omega$. This assumption is significantly weaker than previous ones, for example, we have not required that $a_{1}$ is not strongly decreasing for $x$, cf., $[11,21]$.

\subsection{Accurate estimates for problems 1 and 2}

As we have seen, $\gamma h^{-1}$ is a bound of the condition number of the preconditioned matrix. In some cases, however, the constant $\gamma$ given by (30) may be very large if the coefficient $a_{1}$ oscillates very frequently in $x$-direction or if $a_{1}(x, y) / a_{1}(z, y)$ is very large for some $x, z \in(0, a), x<z$. In this subsection we show how to use the results in Sect. 3 to obtain more accurate bounds for the condition number for the problems 1 and 2. Recalling Theorem 2, we know that the condition number of the preconditioned matrix is dominated by the spectral radii $\rho\left(D_{i}^{-1} A_{i i}\right)$ and $\rho\left(M_{i i}\right)$. Our aim is to estimate $\rho\left(D_{i}^{-1} A_{i i}\right)$ and $\rho\left(M_{i i}\right)$ more accurately. We assume that there exist two integrable functions $F$ and $G$ over $(0, a)$ such that

$$
G\left(x_{1}\right) \leq \frac{a_{1}\left(x_{1}, y\right)}{a_{1}\left(x_{2}, y\right)} \leq \frac{F\left(x_{1}\right)}{F\left(x_{2}\right)}
$$

for any $x_{1} \leq x_{2}$ in $(0, a)$ and $1 / G, 1 / F$ are also integrable over $(0, a)$.

By induction first we show that

$$
\begin{gathered}
D_{i} \mathbf{e} \geq \alpha_{i}^{-1} \triangle_{i} \mathbf{e}, \\
\alpha_{i}=\left(\sum_{k=0}^{i-1} 1 / G((k-1 / 2) h)\right) /\left(\sum_{k=0}^{i} 1 / G((k-1 / 2) h)\right) .
\end{gathered}
$$

Inequality $D_{1} \mathbf{e}=A_{11} \mathbf{e} \geq\left(\triangle_{1}+\triangle_{0}\right) \mathbf{e}$ implies (32) for $i=1$, where

$$
\triangle_{0}= \begin{cases}\operatorname{diag}\left(a_{1}\left({ }_{2}^{1} h, \delta\right), a_{1}\left({ }_{2}^{1} h, 2 \delta\right), \ldots, a_{1}\left({ }_{2}^{1} h, n \delta\right)\right) & \text { for problem 1, } \\ \operatorname{diag}\left({ }_{2}^{1} a_{1}\left({ }_{2}^{1} h, 0\right), a_{1}\left({ }_{2}^{1} h, \delta\right), \ldots, a_{1}\left({ }_{2}^{1} h,(n-1) \delta\right)\right) & \text { for problem 2. }\end{cases}
$$

It follows from (21) that

$$
D_{i} \mathbf{e}=A_{i i} \mathbf{e}-A_{i, i-1} D_{i}^{-1} A_{i-1, i} \mathbf{e} \geq\left(\triangle_{i}+\triangle_{i-1}\right) \mathbf{e}-\alpha_{i-1} \triangle_{i} \mathbf{e} \geq \alpha_{i}^{-1} \triangle_{i} \mathbf{e}
$$

We are now in a position to estimate the following bound for $\rho\left(D_{i}^{-1} A_{i i}\right)$, 
(33) $\rho\left(D_{i}^{-1} A_{i i}\right) \leq$

$$
\begin{gathered}
\left(1+\frac{F((i-1 / 2) h)}{F((i+1 / 2) h)}\right) \sum_{k=0}^{i-1} 1 / G((k+1 / 2) h) \\
\sum_{k=0}^{i-1} 1 / G((k+1 / 2) h)+\frac{F((i-1 / 2) h)}{F((i+1 / 2) h)} G((i-1 / 2) h)
\end{gathered}
$$

By a direct computation we have

$$
\begin{aligned}
\gamma_{i} D_{i} \mathbf{e} & \geq A_{i i} \mathbf{e}+\left(\gamma_{i}-1\right) A_{i i} \mathbf{e}-\gamma_{i} A_{i, i-1} D_{i-1}^{-1} A_{i-1, i} \mathbf{e} \\
& \geq A_{i i} \mathbf{e}+\left(\gamma_{i}-1\right)\left(\triangle_{i}+\triangle_{i-1}\right) \mathbf{e}-\gamma_{i} \frac{\sum_{k=0}^{i-2} 1 / G((k+1 / 2) h)}{\sum_{k=0}^{i-1} 1 / G((k+1 / 2) h)} \mathbf{e} \\
& \geq A_{i i} \mathbf{e}+\triangle_{i-1}\left(\left(\gamma_{i}-1\right) \triangle_{i} \triangle_{i-1}^{-1} \mathbf{e}-\gamma_{i} \frac{\sum_{k=0}^{i-2} 1 / G((k+1 / 2) h)}{\sum_{k=0}^{i-1} 1 / G((k+1 / 2) h)} \mathbf{e}\right) \\
& \geq A_{i i} \mathbf{e}+\triangle_{i-1}\left(\left(\gamma_{i}-1\right) \frac{F((i+1 / 2) h)}{F((i-1 / 2) h)}-\gamma_{i} \sum_{k=0}^{i-2} 1 / G((k+1 / 2) h)\right. \\
& \geq A_{i i} \mathbf{e},
\end{aligned}
$$

which implies (33) because $\gamma_{i} D_{i}-A_{i i}$ is a Z-matrix as mentioned in Subsect. 4.1. Furthermore, $\gamma_{i}$ can be estimated as follows:

$$
\gamma_{i} \approx \frac{\left(1+\frac{F((i-1 / 2) h)}{F((i+1 / 2) h)}\right) \int_{0}^{i h} \frac{d x}{G(x)}}{\int_{0}^{i h} \frac{d x}{G(x)}+\frac{F((i-1 / 2) h)}{F((i+1 / 2) h)} G((i-1 / 2) h)} .
$$

Again, if $a_{1}(x, y)$ is non-decreasing for $x$, it follows straightforwardly from (33) that

$$
\rho\left(D_{i}^{-1} A_{i i}\right) \leq \sum_{k=0}^{\left.2 \sum_{k=0}^{i-1} 1 / G(k+1 / 2) h\right)}<2 .
$$

Applying Corollary 1 shows that

$$
\begin{gathered}
\lambda_{n(m-i)+j}\left(C^{-1} A_{i i}\right) \leq \frac{G((i-1 / 2) h)}{2} \sum_{k=0}^{i-1} 1 / G((k+1 / 2) h)+\frac{1}{2}, \\
i=1,2, \ldots, m, \quad j=1,2, \ldots, n .
\end{gathered}
$$

It follows from (32) that $D_{i}^{-1} \triangle_{i} \mathbf{e} \leq \alpha_{i} \mathbf{e}$ and

$$
\begin{aligned}
D_{i}^{-1} \triangle_{i-1} \mathbf{e} & \leq D_{i}^{-1} \triangle_{i} \triangle_{i-1} \triangle_{i}^{-1} \mathbf{e} \\
& \leq D_{i}^{-1} \triangle_{i} \frac{F((i-1 / 2) h)}{F((i+1 / 2) h)} \mathbf{e} \leq \alpha_{i} F((i-1 / 2) h) \mathbf{F}
\end{aligned}
$$


Hence,

$$
\begin{aligned}
& D_{i}^{-1 / 2} T_{k i}^{\mathrm{T}} T_{k i} D_{i}^{1 / 2} \mathbf{e}=\left(D_{i}^{-1} \triangle_{i}\right) \cdots\left(D_{k-1}^{-1} \triangle_{k-1}\right)\left(D_{k}^{-1} \triangle_{k-1}\right) \cdots\left(D_{i+1}^{-1} \triangle_{i}\right) \mathbf{e} \\
& \leq \sum_{j=0}^{i-1} 1 / G((j+1 / 2) h) \sum_{j=0}^{i} 1 / G((j+1 / 2) h) \\
& \sum_{j=0}^{k-1} 1 / G((j+1 / 2) h) \sum_{j=0}^{k} 1 / G((j+1 / 2) h)
\end{aligned}
$$

which implies that the spectral radius satisfies

$$
\begin{aligned}
\rho\left(T_{i k} T_{i k}^{\mathrm{T}}\right)= & \rho\left(D_{i}^{-1 / 2} T_{i k} T_{i k}^{\mathrm{T}} D_{i}^{1 / 2}\right) \\
& \sum_{j=0}^{i-1} 1 / G((j+1 / 2) h) \sum_{j=0}^{i} 1 / G((j+1 / 2) h) F((i+1 / 2) h), \\
\leq & \sum_{j=0}^{k-1} 1 / G((j+1 / 2) h) \sum_{j=0}^{k} 1 / G((j+1 / 2) h)^{F((k+1 / 2) h)},
\end{aligned}
$$

due to the nonnegativity of $D_{i}^{-1 / 2} T_{k i}^{\mathrm{T}} T_{k i} D_{i}^{1 / 2}$. Therefore,

$$
\begin{aligned}
& \rho\left(M_{k k}\right)=\rho\left(\sum_{k=i}^{m} T_{k i}^{\mathrm{T}} T_{k i}\right) \leq \sum_{k=i}^{m} \rho\left(T_{k i}^{\mathrm{T}} T_{k i}\right) \\
\leq & F((i+1 / 2) h) \sum_{j=0}^{i-1} 1 / G((j+1 / 2) h) \sum_{j=0}^{i} 1 / G((j+1 / 2) h) \\
& \sum_{k=i}^{m}\left(\frac{1}{F((k+1 / 2) h) \sum_{j=0}^{k-1} 1 / G((j+1 / 2) h) \sum_{j=0}^{k} 1 / G((j+1 / 2) h)}\right) .
\end{aligned}
$$

Similarly, by definition of integration, $\rho\left(M_{i i}\right)$ can be estimated by

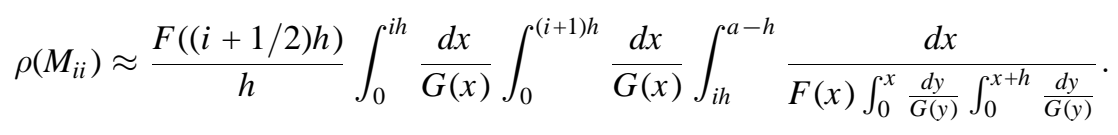

\subsection{Examples}

In this subsection we present three particular examples by using the results from the previous subsections. For all examples $a=b=1$.

Example 1. First we consider a variable coefficient $a_{1}(x, y)=1+|x-1 / 2|^{\alpha} y$, where $\alpha$ is a positive constant. It follows straightforwardly from our analysis that

$$
F(x)= \begin{cases}1+(1 / 2-x)^{\alpha}, & \text { if } x \leq 1 / 2 \\ 1, & \text { if } x \geq 1 / 2\end{cases}
$$


satisfies condition (25). Using (29) with a simple estimate of integration shows that

$$
\lambda_{\max }\left(C^{-1} A\right) \leq\left(2\left(\frac{1}{2}+\frac{1}{(\alpha+1) 2^{\alpha+1}}\right)^{\frac{1}{2}}+\frac{1}{2^{\alpha}}\right) h^{-1}+o\left(h^{-1}\right) .
$$

Example 2. We consider an example with a discontinuous coefficient

$$
a_{1}(x, y)= \begin{cases}d, & (x, y) \in\left(\frac{1}{2}, 1\right) \times\left(\frac{1}{2}, 1\right), \\ 1, & \text { otherwise }\end{cases}
$$

If $d \geq 1$ the functions

$$
F(x)=1, \quad G(x)= \begin{cases}d, & x \in\left(\frac{1}{2}, 1\right), \\ 1 & \text { otherwise }\end{cases}
$$

satisfy condition (31), and if $d<1$ the functions

$$
F(x)=\left\{\begin{array}{cc}
d, & x \in\left(\frac{1}{2}, 1\right), \\
1 & \text { otherwise, }
\end{array} \quad G(x)=1\right.
$$

satisfy condition (31). We first consider problem 3. It follows from (23) and (28) that

$$
\rho\left(D_{1} A_{11}\right)=0, \quad \rho\left(D_{i}^{-1} A_{i i}\right) \leq 1+\frac{F((i-3 / 2) h)}{F((i-1 / 2) h)}, i \geq 2, \quad \rho\left(M_{j_{0}, j_{0}}\right) \leq \frac{m}{2}+1,
$$

where $j_{0}=\left\lceil\begin{array}{c}m \\ 2\end{array}\right\rceil+1$ and $\lceil x\rceil$ denotes the integer ceiling function of $x$. Applying Theorem 3 shows that

$$
\lambda_{\max }\left(C^{-1} A\right) \leq \begin{cases}\frac{d^{-1}+1}{2} m, & \text { if } d<1 \\ m, & \text { if } d \geq 1\end{cases}
$$
bound

For problems 1 and 2 we consider $d \geq 1$ only. Inequality (33) gives the

$$
\rho\left(D_{i}^{-1} A_{i i}\right) \leq \begin{cases}2 i & i \leq\left\lceil\begin{array}{c}
m \\
2
\end{array}\right\rceil, \\
i+1, & 2\left(\left\lceil_{2}^{m}\right\rceil+\left(i-\left\lceil_{2}^{m}\right\rceil\right) d^{-1}\right) \\
\left\lceil\begin{array}{c}
m \\
2
\end{array}+\left(i+1-\left\lceil_{2}^{m}\right\rceil\right) d^{-1}\right. & i>\left\lceil\begin{array}{c}
m \\
2
\end{array}\right\rceil\end{cases}
$$

and (28) yields

$$
\rho\left(M_{i i}\right) \leq m-i+1 .
$$

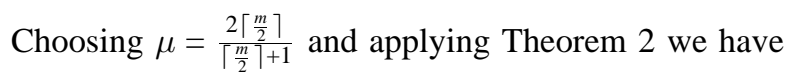




$$
\begin{aligned}
\lambda_{\max }\left(C^{-1} A\right) & \leq \frac{\left\lceil\begin{array}{c}
m \\
2
\end{array}\right\rceil+1}{2}+\sum_{i=\left\lceil\begin{array}{l}
m \\
2
\end{array}\right\rceil+1}^{m}\left(\rho\left(D_{i}^{-1} A_{i i}\right)-\mu\right) \rho\left(M_{i i}\right) \\
& \leq \frac{\left\lceil\begin{array}{c}
m \\
2
\end{array}\right\rceil+1}{2}+\sum_{i=\left\lceil^{m}\right\rceil+1}^{m}(2-\mu)(m-i+1) \\
& \leq \frac{3}{4} m+\frac{1}{2} .
\end{aligned}
$$

Also we can easily derive a simple bound for $d<1$ similarly to what we did for $d \geq 1$, but we prefer to do first a transformation $x \mapsto 1-x$. Then the upper bound given by (37) is valid for the maximum eigenvalue of the corresponding preconditioned matrix. If $d=1$ the result can be further improved. In fact, Inequality (35) becomes

$$
\rho\left(M_{i i}\right) \leq i(i+1) \sum_{k=i}^{m} \frac{1}{k(k+1)}=i(i+1)\left(\frac{1}{i}-\frac{1}{m+1}\right)=\frac{(i+1)(m+1-i)}{m+1} .
$$

Let $p=\lceil 0.54(m+1)\rceil-1$ and $\mu=\frac{2 p}{p+1}$. Using Theorem 2 again shows that

$$
\text { (38) } \begin{aligned}
\lambda_{\max }\left(C^{-1} A\right) & \leq \frac{p+1}{2}+\sum_{i=p+1}^{m}\left(\begin{array}{c}
2 i \\
i+1
\end{array}-\frac{2 p}{p+1}\right) \frac{(i+1)(m+1-i)}{m+1} \\
& =\frac{p+1}{2}+\frac{2}{(p+1)(m+1)} \sum_{i=p+1}^{m}(i-p)(m+1-i) \\
& \leq \frac{p+1}{2}+\frac{2 m^{3}}{(p+1)(m+1)} \int_{\substack{p \\
m}}^{1}\left(x-\frac{p}{m}\right)\left(1+\frac{1}{m}-x\right) d x \\
& \leq p+1+\frac{(m-p)^{2}}{2}+\frac{1}{(m+1)(p+1)}+\frac{(m-p)^{3}}{3(m+1)(p+1)} \\
& \leq \frac{\Gamma 0.54(m+1)\rceil}{2}+\frac{\lfloor 0.46(m+1)\rfloor^{2}}{2}+\frac{1}{3}[0.46(m+1)\rfloor^{3} \\
& \leq 0.3301(m+1)+0.5 .
\end{aligned}
$$

Example 3. As the third example we consider a typical problem

$$
a_{1}(x, y)= \begin{cases}d, & \text { if }(x, y) \in\left(\begin{array}{l}
1 \\
4
\end{array}, \frac{3}{4}\right) \times\left(\begin{array}{l}
1 \\
4
\end{array}, \frac{3}{4}\right), \\
1, & \text { otherwise }\end{cases}
$$

for problem 3 (from $[12,19,24]$ ) and numerical comparisons, where $d$ is a positive constant.

By using Theorem 3 it follows in a similar way as for Example 2 that

$$
\lambda_{\max }\left(C^{-1} A\right) \leq\left\{\begin{array}{cl}
\frac{d+3}{4} m, & \text { if } d \geq 1 \\
\frac{1+3 d^{-1}}{4} m, & \text { if } d<1
\end{array}\right.
$$


The following table shows the values of the exact maximum eigenvalues of the preconditioned matrix $C^{-1} A$ for different $h$ and $d=10^{k}$.

Table 1. Numerical results of the exact maximum eigenvalues for Example 3

\begin{tabular}{ccccccc} 
& \multicolumn{6}{c}{$\lambda_{\max }\left(C^{-1} A\right)$} \\
$h^{-1}$ & $k=-3$ & $k=-2$ & $k=-1$ & $k=1$ & $k=2$ & $k=3$ \\
20 & 6.2427 & 7.7367 & 6.8336 & 6.7593 & 8.9943 & 7.8979 \\
40 & 22.328 & 35.651 & 16.943 & 16.400 & 37.985 & 26.625 \\
80 & 135.41 & 138.07 & 38.026 & 37.921 & 138.14 & 145.93 \\
160 & 845.12 & 408.18 & 86.967 & 86.884 & 409.61 & 855.82 \\
320 & 2884.8 & 1138.1 & 190.77 & 190.71 & 1138.1 & 2905.5
\end{tabular}

Let $\lambda_{\max }(h, d)$ denote the maximum eigenvalue of the preconditioned matrix $C^{-1} A$ given in Table 1 with respect to the parameters $h$ and $d$ for Example 3 and let $\widetilde{\lambda}_{\max }(h, d)$ denote the upper bound of $\lambda_{\max }(h, d)$ given by (39). In some cases the upper bound $\widetilde{\lambda}_{\max }(h, d)$ can be much larger than the actual maximum eigenvalue $\lambda_{\max }(h, d)$ if $h^{-1}$ is small, but for a fixed $d$ the rate $\widetilde{\lambda}_{\max }(h, d) / \lambda_{\max }(h, d)$ decreases rapidly as $h^{-1}$ increases. For all possible values of $h$ and $d$ in Table $1 \min _{h, d} \widetilde{\lambda}_{\max }(h, d) / \lambda_{\max }(h, d)=5.4533$. Therefore, the estimates of upper bounds of the maximum eigenvalue of the preconditioned matrix $C^{-1} A$ are quite reasonable.

Finally, note that no previous result is applicable to Example 1 for $\alpha<1$ and to Example 3 for $d<1$ to get an $O\left(h^{-1}\right)$ upper bound. For the other cases the estimates here are more accurate than the previous ones found in [7] and [18].

Acknowledgements. We are grateful to Magolu Monga-Made for help in numerical computations. The first author is grateful to Gene Golub for the information on incomplete factorizations.

\section{References}

1. Axelsson, O. (1976): A class of iterative methods for finite element equations. Comput. Methods Appl. Mech. Engrg. 9, 123-137

2. Axelsson, O. (1977): Solution of linear systems of equations: iterative methods. In Barker, V. A ed., Sparse Matrix Techniques. Lecture Notes in Mathematics 572, Springer Verlag, Berlin, Heidelberg, New York, 1-50

3. Axelsson, O. (1992): Bounds of eigenvalues of preconditioned matrices. SIAM J. Matrix Anal. Appl. 13, 847-862

4. Axelsson, O. (1994): Iterative Solution Methods. Cambridge University Press, New York

5. Axelsson, O., Brinkkemper, S., Il'in, V. P. (1984): On some versions of incomplete block-matrix factorization methods. Linear Algebra Appl. 38, 3-15

6. Axelsson, O., Lindskog, G. (1986): On the rate of convergence of the preconditioned conjugate gradient method. Numer. Math. 48, 499-523

7. Axelsson, O., Lu, H. (1995): On eigenvalue estimates for block incomplete factorization methods. SIAM J. Matrix Anal. Appl. 16, 1074-1085

8. Beauwens, R. (1984): Upper eigenvalue bounds for pencils of matrices. Linear Algebra Appl. 62, $87-104$

9. Beauwens, R. (1989): Approximate factorizations with S/P consistently ordered M-factors. BIT 29, 658-681 
10. Beauwens, R. (1994): Approximate factorizations with modified S/P consistently ordered Mfactors. Numer. Linear Algebra Appl. 1, 3-17

11. Beauwens, R., Bouzid, M. (1988):. Existence and conditioning properties of sparse approximate block factorizations. SIAM J. Numer. Anal. 25, 941-956

12. Concus, P., Golub G. H., Meurant, G. (1985): Block preconditioning for the conjugate gradient method. SIAM J. Sci. Stat. Comput. 6, 220-252

13. Concus, P., Golub, G. H., O'Leary, D. (1976): A generalized conjugate gradient method for numerical solution of elliptic partial differential equations. In Bunch, J. R. and Rose, D.J. eds., Sparse Matrix Computations, Academic Press, New York, 309-332

14. Greenbaum, A. (1979): Comparison of splittings used with the conjugate gradient algorithm. Numer. Math. 33 181-194

15. Horn, R. A., Johnson, C. R. (1991): Topics in Matrix Analysis. Cambridge University Press, New York

16. Jennings, A. (1977): Influence of the eigenvalues spectrum on the convergence rate of the conjugate gradient method. IMA J. Numer. Anal. 20, 61-72

17. Lu, H. (1995): Forward-backward heat equations and analysis of iterative methods. PhD thesis, Department of Mathematics, University of Nijmegen, The Netherlands

18. Magolu, M. M. (1993): Analytical bounds for block approximate factorization methods. Linear Algebra Appl. 179, 33-57

19. Magolu, M. M. (1995) Ordering strategies for modified block incomplete factorizations. SIAM J. Sci. Comput. 16, 378-399

20. Magolu, M. M., Notay, Y. (1991): On the conditioning analysis of block approximate factorization methods. Linear Algebra Appl. 154-156, 583-599

21. Notay, Y. (1991): Conditioning analysis of modified block incomplete factorizations. Linear Algebra Appl. 154-156, 711-722

22. Parlett, B. N. (1980): The Symmetric Eigenvalue Problem. Prentice-Hall, Englewood Cliffs, N.J.

23. Van der Sluis, A., Van der Vorst, H. A. (1986): The rate of convergence of conjugate gradients. Numer. Math. 48, 543-560

24. Van der Vorst, H. A. (1990): The convergence behavior of preconditioned CG and CG-S. In O. Axelsson and L. Kolotilina, eds, Preconditioned Conjugate Gradient Methods, Lecture Notes in Mathematics 1457, Springer Verlag, Berlin, 126-136

This article was processed by the author using the $\mathrm{LAT}_{\mathrm{E}} \mathrm{X}$ style file pljour $1 m$ from Springer-Verlag.

Numerische Mathematik Electronic Edition

page 209 of Numer. Math. (1997) 78: 189-209 